\title{
Performance of the Tracking System at the LHCb Experiment
}

\section{Silvia Borghi ${ }^{* \dagger}$}

Department of Physics and Astronomy, University of Glasgow, Glasgow, G12 8QQ, UK

E-mail:silvia.borghi@cern.ch

The $\mathrm{LHCb}$ experiment is specialized in a very precise reconstruction of B decays into many particle final states. The $\mathrm{LHCb}$ detector is a forward spectrometer with a tracking system based on silicon strip detectors and straw-tube drift chambers before and after a dipole generating a magnetic field, to precisely measure the vertex position and the momentum resolution of the particles traveling through the detector. We report on the performances of the individual tracking subdetectors in terms of hit resolution and detector efficiencies, as well as on the overall track reconstruction performance and the alignment status.

35th International Conference of High Energy Physics - ICHEP2010,

July 22-28, 2010

Paris France

\footnotetext{
${ }^{*}$ Speaker.

$\dagger$ On behalf of the LHCb Collaboration.
} 


\section{Introduction}

The LHCb experiment [1] is dedicated to the study of heavy flavour physics at LHC. Its primary goal is to look for indirect evidence of new physics in CP violation and rare decays of beauty and charm hadrons. As b $\bar{b}$ pairs are produced mainly close to the beam direction at LHC energy, the LHCb detector is a forward spectrometer with a $\eta$ acceptance between 1.9 and 4.9. One essential element for $\mathrm{LHCb}$ physics goals is a high precision in the tracking to have an excellent vertex resolution and high momentum measurement precision. The tracking system consist of silicon strip detectors and straw-tube drift chambers. The experiment has a PID system composed of two RICH detectors for the kaon and pion separation in the energy range between 2 and $100 \mathrm{GeV}$, and of an electromagnetic and hadron calorimeter followed by the muon stations (more details can be found in these proceeding [2]).

\section{Detector description}

The vertex detector (VELO) is composed of 21 silicon micro-strip stations with and R- $\Phi$ geometry perpendicular to the beam direction with a strip pitch between 40 and $100 \mu \mathrm{m}$. The sensors are placed at a radial distance of $7 \mathrm{~mm}$ from the beam, which is smaller than the aperture required by the LHC during beam injection and machine developments. Therefore the detector consists of two equal halves that can be retracted at a distance of $30 \mathrm{~mm}$ from the beam for each LHC injection and inserted when stable beam is declared. All the sensors of the VELO are powered and working properly. The cluster finding efficiency is measured to be $99.8 \%$. The best hit resolution measured is $4 \mu \mathrm{m}$ for a strip pitch of $40 \mu \mathrm{m}$ for the optimal angle track. This is in good agreement with the Monte Carlo considering the current sensor alignment. More details can be found in these proceeding [3].

The tracker system is composed of a silicon micro-strip detector (TT) upstream the magnet, and three tracking stations downstream the magnet, composed of a silicon micro-strip detector in the inner parts (IT) and of straw-tubes for the outer parts (OT). The TT is composed of four layers of silicon micro-strip sensors with a pitch of $183 \mu \mathrm{m}$ and covers the full acceptance of the experiment. In the two central layers each module is rotated by a stereo angle of \pm 5 degrees. Each IT station consists of four individual detector boxes arranged around the beam pipe. Each box contains four detection layers of silicon micro-strip with a strip pitch of $198 \mu \mathrm{m}$. The two central layers are rotated by a stereo angle of \pm 5 degrees. $99.6 \%$ of the TT detector channels and $98.6 \%$ of the IT channels are functional. For both detectors, the measured cluster finding efficiency was evaluated using high momentum isolated tracks to minimize the effect of ghosts tracks. An efficiency of $99.3 \%$ for the TT and $99.8 \%$ for IT was measured. The single hit resolution is evaluated by the unbiased residual distribution. The resolution is $55 \mu \mathrm{m}$ for TT and $54 \mu \mathrm{m}$ for IT. It is in good agreement with the Monte Carlo simulations considering the current alignment accuracy, that is 35 $\mu \mathrm{m}$ for TT and $16 \mu \mathrm{m}$ for IT.

The Outer Tracker (OT) surrounds the IT detector to cover completely the acceptance of LHCb. It is composed of an array of straw-tubes drift chambers with an inner diameter of about $4.9 \mathrm{~mm}$. The gas mixture is composed of Argon (70\%), $\mathrm{CO}_{2}(28.5 \%)$ and $\mathrm{O}_{2}(1.5 \%)$. As for IT detector, the modules are arranged in three stations and each station consists of four layers. The two central 

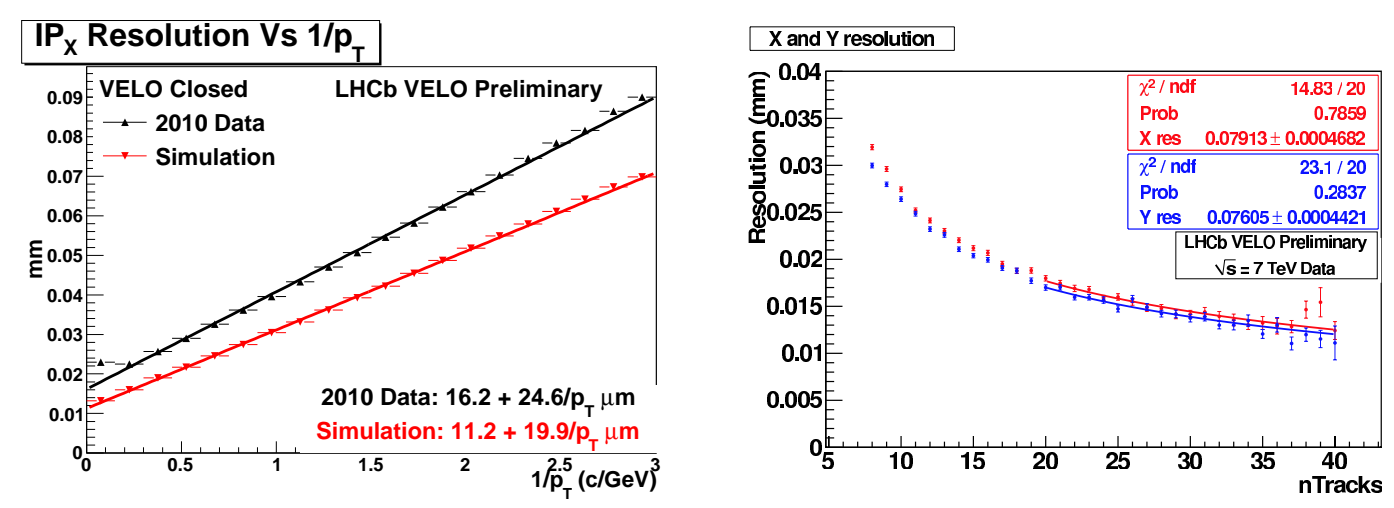

Figure 1: The left hand histogram shows the $\mathrm{IP}_{X}$ resolution as function of $1 / p_{T}$. The right hand histogram shows the primary vertex resolution as function of track multiplicty. The data points are fitted between 20 and 40 tracks with the function $\sigma_{\mathrm{res}}=\sigma_{0} / \sqrt{N}$, where $\sigma_{0}$ is the fitted constant and $\mathrm{N}$ is the number of tracks.

layers are tilted by \pm 5 degrees with respect to the vertical. The $99.3 \%$ of channels are working properly. The single cell efficiency is $98.7 \%$ and it is uniform with respect to the space position. The behaviour is in good agreement with the expectation as measured in the test beam. The hit resolution is measured to be $250 \mu \mathrm{m}$ and it is close to the nominal one.

\section{Detector Performance}

The reconstruction of the primary vertices (PV) and decay vertices of beauty and charm hadrons is essential to provide an accurate measurement of the decay lifetimes and to measure the impact parameter of particles used to tag their flavour.

Assuming that all tracks are originated from primary interation, the IP resolution is measured as the spread of its distribution in bins of $1 / p_{T}$. It increases linearly as function of the inverse of the transverse momentum and it is shown in fig. 1. The linear dependence can be parametrized for the $x$ and $y$ components as $\sigma_{I P_{x}}=16.2 \mu \mathrm{m}+24.6 \mu \mathrm{m} / p_{T}$ and $\sigma_{I P_{y}}=15.7 \mu \mathrm{m}+24.4 \mu \mathrm{m} / p_{T}$ with $p_{T}$ in $\mathrm{GeV} / \mathrm{c}$. The difference between measured IP resolution and the one evaluated on Monte Carlo data is expected to be reduced thanks to better alignment and better material description.

The primary vertex resolution is strongly correlated to the number of tracks making the vertex, the track multiplicity. The PV resolution can be measured by randomly splitting the track sample in each event in two sets. The resolution (shown in fig. 1) is extracted comparing the two vertices with the same track multiplicity. The method was validated on MC data. The preliminary resolution of a PV composed of 25 tracks is $(15.8,15.2,91) \mu \mathrm{m}$ for the $(x, y, z)$ components.

An excellent momentum resolution is an essential prerequisite for the good invariant mass resolution, particularly for two-body decays, which leads to higher sensitivity in rare decay searches as well as to lower background levels in general. In LHCb, a very good mass resolution was obtained, for example we measure a resolution of $3.3 \mathrm{MeV}$ for $K_{s} \rightarrow \pi \pi$ and $9 \mathrm{MeV}$ for $D^{0} \rightarrow K \pi$.

The reconstruction of high multiplicity of beauty and charm decays demands a high tracking efficiency. Several methods to evaluate the efficiency from the data were developed. Mainly we use a tag-and-probe method. One method, using $K_{s} \rightarrow \pi \pi$, selects events where the 'tag' pion is reconstructed completely by the tracking system, while the other 'probe' pion is reconstructed 

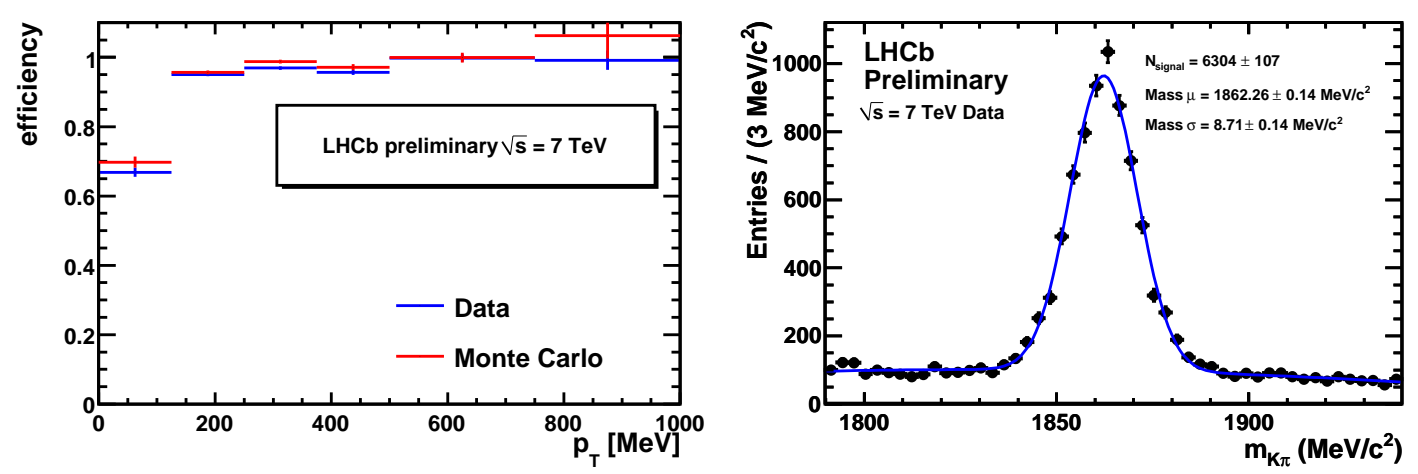

Figure 2: The left hand histogram shows the efficiency as function of $p_{T}$ for data (blue) and for Monte Carlo (red). The right hand histogram shows the mass invariant for $D^{0} \rightarrow K \pi$.

using only a VELO track segment matched with a cluster in the calorimeter. One can evaluate the efficiency of the T station checking the matching of 'probe' track with the segment in the T station. The measured efficiency as a function of the transverse momentum is shown in fig. 2. It is about $95 \%$ for all tracks with a transverse momentum above $100 \mathrm{MeV} / \mathrm{c}$ which covers the full physics range of the experiment. Similar probe-and-tag method is used to evaluate the VELO efficiency. The same methods can be also applied to other decays, i.e. $J / \Psi \rightarrow \mu \mu$.

An additional cross-check to evaluate the efficiency uses some well known physics channels. In particular, the efficiency is evaluated by the relative rates of 2-prong decay $D^{0} \rightarrow K \pi$ versus 4-prong decay $D^{0} \rightarrow K 3 \pi$, that are known at $2 \%$ level. The ratio between the efficiency obtained with the data and $\mathrm{MC}$ was evaluated to be:

$$
\varepsilon(\text { track }) \sim \sqrt{\frac{N(K \pi \pi \pi)}{N(K \pi)} \cdot \frac{B R(K \pi)}{B R(K \pi \pi \pi)}}=1.03 \pm 0.03
$$

The results of these methods show an agreement of the efficiency between data and MC within their uncertainties of $3-4 \%$.

\section{Conclusion}

LHCb is an experiment dedicated to the study of the heavy flavours and the tracking system performances are of crucial importance for the physics results. The tracking is fully operational, with good performances, both in resolution and efficiency evaluated using $\sqrt{s}=7 \mathrm{TeV}$ collision data.

\section{References}

[1] The LHCb Collaboration, The LHCb Detector at the LHC, J. Instrum. 3 (2008) S08005.

[2] A. Powell these proceedings.

[3] S. Borghi these proceedings. 